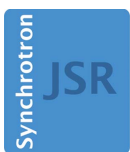

JOURNAL OF SYNCHROTRON RADIATION

ISSN $1600-5775$

Received 11 March 2017

Accepted 19 January 2018

Edited by I. Lindau, Stanford University, USA

Keywords: resonant inelastic X-ray scattering; X-ray emission; ID20 beamline; RIXS spectrometer; ESRF.

\section{A high-energy-resolution resonant inelastic X-ray scattering spectrometer at ID20 of the European Synchrotron Radiation Facility}

\author{
M. Moretti Sala, ${ }^{a} *$ K. Martel, ${ }^{a}$ C. Henriquet, ${ }^{a}$ A. Al Zein, ${ }^{a, b}$ L. Simonelli, ${ }^{a, c}$ \\ Ch. J. Sahle, ${ }^{a}$ H. Gonzalez, ${ }^{a}$ M.-C. Lagier, ${ }^{a}$ C. Ponchut, ${ }^{a}$ S. Huotari, ${ }^{a, d}$ R. Verbeni, \\ M. Krisch ${ }^{\mathrm{a}}$ and G. Monaco ${ }^{\mathrm{a}, \mathrm{e}}$
}

${ }^{\mathbf{a}}$ ESRF, The European Synchrotron, 71 Avenue des Martyrs, F-38000 Grenoble, France, ${ }^{\mathbf{b}}$ Department of Physics, Faculty of Science, Beirut Arab University, Beirut 11072809, Lebanon, 'ALBA Synchrotron Light Facility, Carrer de la Llum 2-26, 08290 Cerdanyola del Valleés, Barcelona, Spain, ${ }^{\mathbf{d}}$ Department of Physics, University of Helsinki, PO Box 64, FI-00014 Helsinki, Finland, and ${ }^{\mathbf{e}}$ Dipartimento di Fisica, Università di Trento, via Sommarive 14, 38123 Povo (TN), Italy. *Correspondence e-mail: marco.moretti@esrf.fr

An end-station for resonant inelastic X-ray scattering and (resonant) X-ray emission spectroscopy at beamline ID20 of ESRF - The European Synchrotron is presented. The spectrometer hosts five crystal analysers in Rowland geometry for large solid angle collection and is mounted on a rotatable arm for scattering in both the horizontal and vertical planes. The spectrometer is optimized for high-energy-resolution applications, including partial fluorescence yield or highenergy-resolution fluorescence detected X-ray absorption spectroscopy and the study of elementary electronic excitations in solids. In addition, it can be used for non-resonant inelastic X-ray scattering measurements of valence electron excitations.

\section{Introduction}

Beamline ID20 is the state-of-the-art inelastic X-ray scattering (IXS) beamline of ESRF - The European Synchrotron (Grenoble, France). This beamline evolved from one of the two branches of the previous ID16 beamline as the UPBL6 project in the frame of the ESRF Upgrade - Phase I. ID20 is today fully dedicated to the study of the electronic structure of materials and provides two branches, optimized for high detection efficiency and high-energy-resolution applications, respectively. The beamline optics common to all its branches will be described elsewhere. The high-efficiency mediumenergy-resolution spectrometer that has been conceived for non-resonant IXS from core levels has been described elsewhere (Huotari et al., 2017). In this article, we describe a highenergy-resolution spectrometer that is mostly dedicated to resonant IXS (RIXS) and (resonant) X-ray emission spectroscopy [(R)XES], but it can also be employed in non-resonant IXS experiments of valence excitations requiring high energy resolution (Larson et al., 2007; Cudazzo et al., 2014). The spectrometer is named RIXS, but formally there is no distinction between RIXS and RXES, and sometimes the two terms are used interchangeably.

RIXS is a photon-in-photon-out technique for the study of the electronic structure of materials as it probes transitions involving lattice, charge, spin and/or orbital degrees of freedom (Kotani \& Shin, 2001; Ament et al., 2011a). In the specific case of low-energy elementary excitations, recent advances in the instrumentation, both in the soft (Ghiringhelli 
et al., 2006, 2012) and hard X-ray regimes (Shvyd'ko et al., 2013; Ishii et al., 2013; Moretti Sala et al., 2013) and in the theoretical interpretation of RIXS spectra (Haverkort, 2010; Ament et al., 2011b,c; Igarashi \& Nagao, 2012; Marra et al., 2012, 2013, 2016; van den Brink \& Veenendaal, 2012; Jia et al., 2014, 2016; Moretti Sala et al., 2014a; Kim \& van den Brink, 2015; Devereaux et al., 2016) boosted the technique enormously. The most prominent example is the study of magnetic excitations in $\mathrm{Cu}$ (Braicovich et al., 2010; Guarise et al., 2010; Le Tacon et al., 2011; Ghiringhelli \& Braicovich, 2013; Dean et al., 2012, 2013a,b, 2014; Ishii et al., 2014; Guarise et al., 2014; Lee et al., 2014; Peng et al., 2015; Moser et al., 2015; Minola et al., 2015; Monney et al., 2016; Meyers et al., 2017) and Ir (Kim et al., 2012a,b; Moretti Sala et al., 2014b, 2015; Donnerer et al., 2016; Gretarsson et al., 2016; Clancy et al., 2016; Calder et al., 2016; Liu et al., 2016; Lu et al., 2017; Cao et al., 2017) oxides. As a matter of fact, RIXS can be considered nowadays as a mature technique for studying magnetism in strongly correlated electron systems in a way complementary to other spectroscopies, such as inelastic neutron scattering (INS), $\mathrm{X}$-ray resonant magnetic scattering (XRMS), X-ray magnetic circular dichroism (XMCD) or optical Raman spectroscopy. Other applications of RIXS comprise the study of phonons (Yavaş et al., 2010; Lee et al., 2013; Moser et al., 2015), crystal and ligand field excitations (Ghiringhelli et al., 2004; Moretti Sala et al., 2011), including orbitons (Ulrich et al., 2009; Benckiser et al., 2013; Kim et al., 2014), and charge-transfer transitions (Kim et al., 2004; Lu et al., 2005). RIXS spectrometers are also employed to isolate element-specific X-ray emission lines in (R)XES, including partial fluorescence yield (PFY) or high-energy-resolution fluorescence-detected (HERFD) X-ray absorption spectroscopy (XAS) studies (Glatzel \& Bergmann, 2005; Glatzel et al., 2009; Bauer, 2014). Finally, RIXS spectrometers can be used to measure resonant elastic X-ray scattering (REXS) with energy resolution of the scattered radiation in order to improve the sensitivity of the technique with respect to conventional energy-integrated detection (Chun et al., 2015).

In a RIXS experiment, an incoming photon is prepared in a state of known energy $\omega_{\mathrm{i}}$, momentum $\mathbf{k}_{\mathrm{i}}$ and polarization $\boldsymbol{\varepsilon}_{\mathrm{i}}$. Upon absorption of the photon, the system undergoes a transition from an initial state, usually the ground state, to a highly excited intermediate state with a hole in a core level; within the lifetime of the core hole, then, the system decays to a lower energy state by emitting a photon. A RIXS spectrometer is then used to analyse the energy $\omega_{\mathrm{o}}$ and momentum $\mathbf{k}_{\mathrm{o}}$ of the scattered photons. Also the scattered-photon polarization can be measured, both in the soft (Braicovich et al., 2014) and hard X-ray regimes (Ishii et al., 2011; Gao et al., 2011). The specific advantages of RIXS reside in the resonant nature of this technique, which naturally provides chemical, site and orbital selectivity. Bulk sensitivity and the possibility to exchange a finite momentum are ensured by the use of X-rays, in particular in the hard energy-range. In core-to-core RXES the intermediate state deep core-hole is filled by the radiative decay of a shallow core-electron under the emission of a photon with characteristic energy. A shallow core-hole is present in the final state of the system and detection of the scattered X-rays should be performed with an energy resolution below its intrinsic lifetime broadening. When the intermediate-state deep core-hole is filled by a valence electron, instead, valence-electron excitations can be studied in the energy-loss range of a few $\mathrm{eV}$ and the process is usually named valence-to-core RXES or sometimes simply RIXS. Energy resolution is therefore a crucial figure of merit for a spectrometer as it defines the energy scale of the lowest accessible excitations.

In this paper, we will present the guidelines that we followed in the design of the hard X-ray RIXS spectrometer installed on beamline ID20 of the ESRF, we will describe in detail its main characteristics and highlight the technical solutions we adopted. Key performance figures are also reported. The reader should be aware that, while most of the figures refer to the most challenging configuration of the spectrometer with the best energy resolution, we will not forget to discuss features that are relevant for other applications, including core-to-core (R)XES.

\section{The multiple-element RIXS spectrometer}

In the hard X-ray energy range, analysis of the scattered photon energy is performed using a Bragg crystal, which typically operates in the so-called Rowland geometry. On ID20, in particular, we adopted a solution based on Johanntype spherical crystal analysers (Johann, 1931), as in the case of several other spectrometers present at the ESRF (Glatzel et al., 2009; Llorens et al., 2012; Kvashnina \& Scheinost, 2016) and elsewhere (Sokaras et al., 2013; Shvyd'ko et al., 2013; Ishii et al., 2013; Duan et al., 2017; Rovezzi et al., 2017). Nevertheless, the new ID20 spectrometer is a rather innovative instrument in several respects. It is installed on a dedicated beamline and has been conceived aiming at (i) maximizing the collection solid angle; (ii) optimizing the energy resolution; (iii) allowing various scattering geometries; in addition to stringent requirements in terms of precision and stability of the mechanical components. In the next subsections, we will detail the points above, while the ID20 beamline will be described elsewhere. It here suffices to mention that the monochromatic beam is focused at the sample position by a Kirkpatrick-Baez (KB) (Kirkpatrick \& Baez, 1948) system down to less than $\sim 20 \mu \mathrm{m} \times 10 \mu \mathrm{m}(\mathrm{H} \times \mathrm{V})$. The spectrometer assembly with its $\mathrm{KB}$ mirrors can be translated into a stand-by position when experiments are to be performed in the downstream hutch with its non-resonant IXS (X-ray Raman spectroscopy) instrument (Huotari et al., 2017). The $\mathrm{X}$-rays are detected by a $5 \times 1$ Maxipix (Ponchut et al., 2011) hybrid pixel detector (500 $\mu \mathrm{m}$ sensor thickness) with $55 \mu \mathrm{m} \times$ $55 \mu \mathrm{m}$ pixel size and $14 \mathrm{~mm} \times 70 \mathrm{~mm}$ active surface area.

\subsection{Collection solid angle}

In the Rowland geometry, diffraction (monochromatization) and focusing of hard X-rays are combined in a single optical element, a spherical crystal analyser. The source (beam 
spot on the sample), crystal analyser and detector must lie on a Rowland circle and their position is Bragg angle $\Theta_{\mathrm{B}}$, i.e. scattered photon energy $\omega_{\mathrm{o}}$, dependent. For convenience, the source of the spectrometer remains fixed in space, while the crystal analysers and detector are displaced to track $\omega_{0}$.

The solid angle of the spectrometer depends on the sourceto-crystal analyser distance, the active surface of the crystal analyser and their number. On beamline ID20, the RIXS spectrometer is equipped with five crystal analysers operating on Rowland circles of either $R=1$ or $2 \mathrm{~m}$ diameter, hosted in dedicated chambers operated in $\mathrm{He}$ atmosphere in order to reduce air absorption and scattering, as shown in Fig. 1. The crystal analysers and detector must simultaneously fulfil the Rowland condition and are therefore equipped with the necessary degrees of freedom. Fig. 2 shows the crystal analysers and detector assembly. The $i$ th crystal analyser unit $(i=0$, $\pm 1, \pm 2)$ is equipped with two translations $\left(x_{i}\right.$ and $\left.z_{i}\right)$ and two rotations ( $\theta_{i}$ and $\chi_{i}$, around the $y_{i}$ and $z_{i}$ axes, respectively) as shown in the inset of Fig. 2. The $x_{i}$ translation stages are directed towards the source and adjacent translations form an angle $\beta$ of $7^{\circ}\left(3.5^{\circ}\right)$ in the $R=1 \mathrm{~m}(2 \mathrm{~m})$ RIXS chamber. The detector unit is mounted on two translation stages $\left(x_{\mathrm{D}}\right.$ and $\left.z_{\mathrm{D}}\right)$ inclined by $21^{\circ}$ with respect to the $x$ and $z$ axes, and can be rotated $\left(\theta_{\mathrm{D}}\right)$ around the $y_{\mathrm{D}} \equiv y$ axis. The angle of inclination has been chosen in order to minimize the stroke of the $x_{\mathrm{D}}$ translation stage. Details of the rotation and translation stages are given in Table 1. In Appendix $A$, we describe how to calculate the trajectories of the crystal analysers and detector in order to fulfil the Rowland condition as a function of the Bragg angle.

The signals from the five crystal analysers are collected simultaneously. In general, separating the foci of the crystal analysers on the detector is recommended as they may have

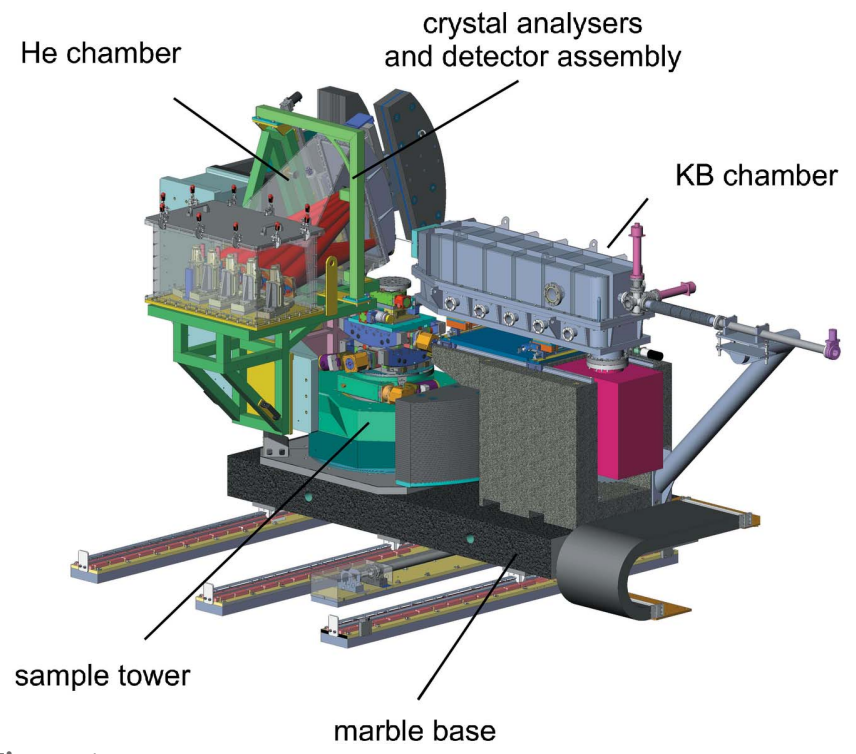

Figure 1

RIXS spectrometer equipped with its $R=1 \mathrm{~m}$ crystal analyser chamber. The KB assembly and the RIXS spectrometer, including the RIXS chamber and the sample tower, sit on a common marble base equipped with a motorized horizontal translation. The whole system is installed on a motorized translation and can be displaced sideways.
Table 1

Details of the crystal analysers and detector rotation and translation stages.

Double values are specific to the $R=1 \mathrm{~m}(2 \mathrm{~m})$ RIXS chambers.

\begin{tabular}{llll}
\hline Name & Description & Stroke & Resolution \\
\hline$\theta_{i}$ & Analyser rotation & $\pm 10^{\circ}$ & $1 \mu \mathrm{rad}$ \\
$\chi_{i}$ & Analyser rotation & $\pm 3^{\circ}$ & $2 \mu \mathrm{rad}$ \\
$x_{i}$ & Analyser translation & $0-102(155) \mathrm{mm}$ & $1 \mu \mathrm{m}$ \\
$z_{\mathrm{i}}$ & Analyser translation & $\pm 12.5 \mathrm{~mm}$ & $1 \mu \mathrm{m}$ \\
$\theta_{\mathrm{D}}$ & Detector rotation & $\pm 20^{\circ}$ & $0.01^{\circ}$ \\
$x_{\mathrm{D}}$ & Detector translation & $0-120 \mathrm{~mm}$ & $0.5 \mu \mathrm{m}$ \\
$z_{\mathrm{D}}$ & Detector translation & $0-640(1270) \mathrm{mm}$ & $1.25 \mu \mathrm{m}$ \\
\hline
\end{tabular}

slightly different energy resolutions and/or calibration, in which case merging them would cause a broadening of the overall energy resolution. For this reason, a two-dimensional detector is used on ID20. Also, a tight selection of the detector 'region of interest' allows reduction of stray-scattering background and the cross-talk between foci that may leak into adjacent chips, as is the case in Fig. 3(c), and, depending on the geometry and scattering angle, discrimination of the signals of the sample and its environment (Huotari et al., 2011). On the other hand, the images of the different crystal analysers can intentionally be superimposed in order to minimize the detector exposure area and thus the stray-scattering background even further. Another case where the use of a twodimensional detector is necessary is in experiments that require momentum resolution, as is often the case when studying electronic elementary excitations in single-crystal samples. Fig. 3 shows typical detector images obtained in various spectrometer configurations, where the signals from the various crystal analysers are focused at the centre of each chip. It should be noted that the use of a two-dimensional detector allows the crystal analyser focusing properties to be assessed; the irregular shape of the foci in Fig. 3(c) is indicative of a poor bent crystal analyers quality.

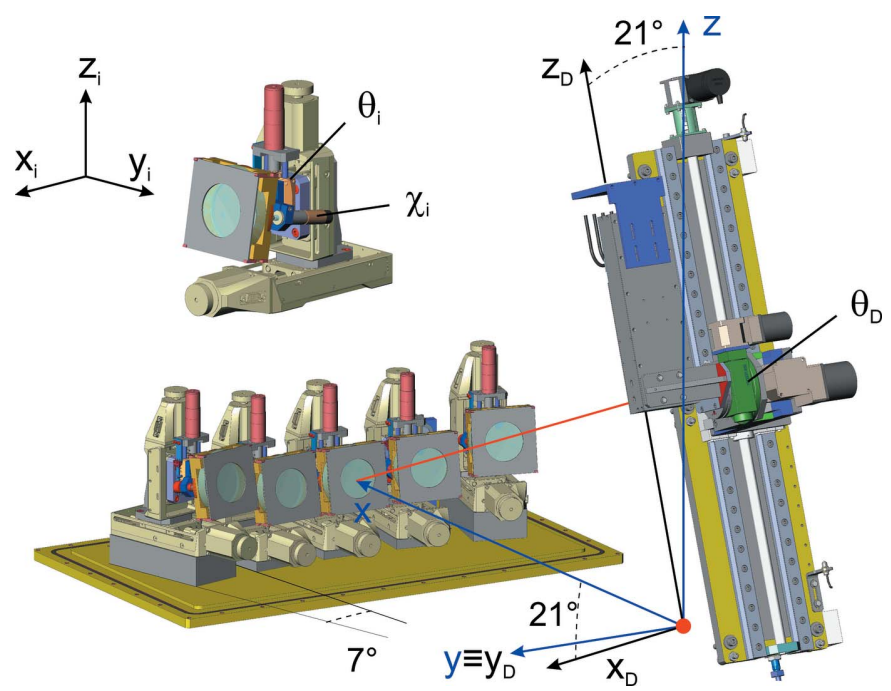

Figure 2

$R=1 \mathrm{~m}$ crystal analysers and detector assembly. The sample position is shown as a red sphere. 
(a)

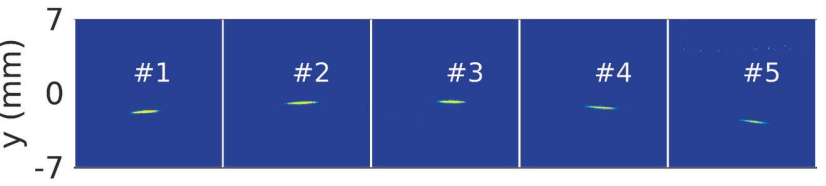

(b)

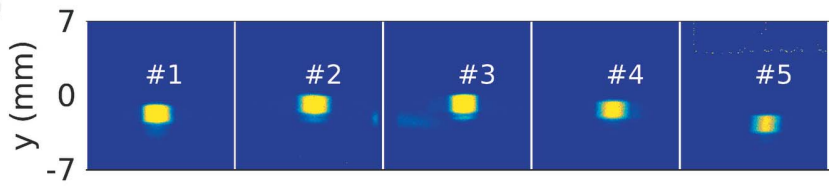

(c)

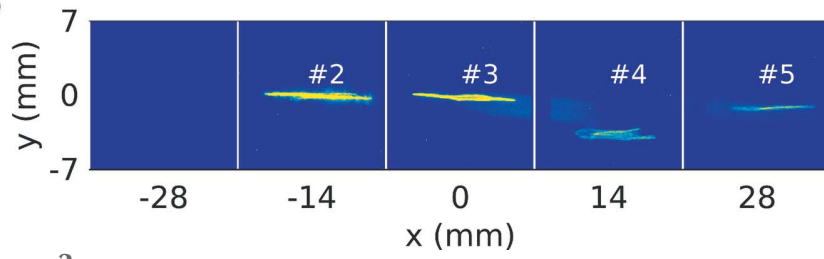

Figure 3

Typical detector images obtained in various spectrometer configurations. When diced crystal analysers are used the focus on the detector is a horizontal straight line $(a)$ if the bandwidth of the incident radiation is much smaller than the cube energy acceptance or a square $(b)$ in the opposite case (Huotari et al., 2005). The size of the square is twice the cube size. When bent crystal analysers are used, instead, the focus is point-like at large Bragg angles, but it becomes astigmatic when the Bragg angle is decreased $(c)$.

\subsection{Energy resolution}

Energy resolution is one of the most important figures of merit of a RIXS spectrometer, as it sets the lowest energy scale of measurable excitations. A number of contributions determines the actual energy resolution of a RIXS spectrometer (Huotari et al., 2006; Moretti Sala et al., 2013), which will be considered in the following.

2.2.1. Analyser contribution. The first contribution to energy resolution is the crystal analyser contribution $\Delta \omega_{\mathrm{a}}$. For high-resolution applications, spherical diced crystal analysers are used (Verbeni et al., 2005). In this case $\Delta \omega_{\mathrm{a}}$ is well approximated by the intrinsic energy resolution of the crystal,

$$
\frac{\Delta \omega_{\mathrm{a}}}{\omega_{\mathrm{o}}}=\frac{\Delta \theta_{\mathrm{D}}}{\tan \Theta_{\mathrm{B}}}\left[\frac{\sin \left(\Theta_{\mathrm{B}}-\alpha\right)}{\sin \left(\Theta_{\mathrm{B}}+\alpha\right)}\right]^{1 / 2},
$$

where $\Theta_{B}$ is the crystal analyser Bragg angle corresponding to the selected photon energy $\omega_{\mathrm{o}}, \Delta \theta_{\mathrm{D}}$ is the Darwin width of the reflection and $\alpha$ is the asymmetry angle. For lower-energyresolution applications, spherically bent crystals are used. The calculation of $\Delta \omega_{\mathrm{a}}$ is more complicated in this case. The energy resolution can be calculated according to

$$
\frac{\Delta \omega_{\mathrm{a}}}{\omega_{\mathrm{o}}}=\frac{t}{R}\left(\frac{1}{\tan ^{2} \Theta_{\mathrm{B}}}-2 v\right),
$$

where $t$ is the absorption length of the material (usually Si or $\mathrm{Ge}$ ) and $v$ is its Poisson's ratio ( $v=0.22$ for $\mathrm{Si}$ and 0.27 for $\mathrm{Ge}$ ). In the symmetric case $(\alpha=0)$, it reduces to $\Delta \omega_{\mathrm{a}} / \omega_{\mathrm{o}}=$ $t / R\left(\cot ^{2} \Theta_{\mathrm{B}}-2 v\right)$. Additional details on the calculation of the energy resolution contribution of bent crystal analysers are given by Honkanen et al. (2014a, 2017).

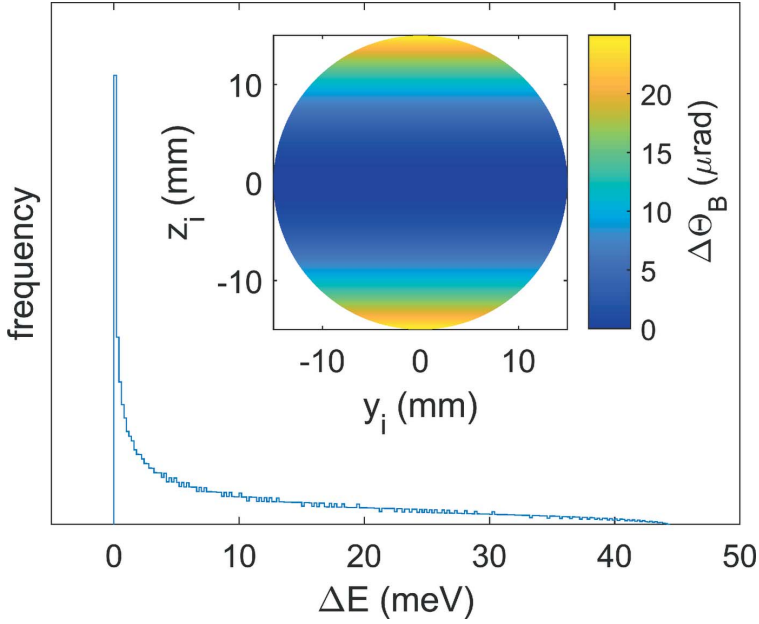

Figure 4

Energy distribution corresponding to the Johann aberration and (inset) variation of the Bragg angle across the surface of a $\mathrm{Si}(551)$ crystal analyser $(R=1 \mathrm{~m})$ with a circular mask of radius $A=15 \mathrm{~mm}$ at the Ni $K$-edge.

2.2.2. Johann aberration. Another contribution to the energy resolution comes from the so-called Johann aberration, which causes the Bragg angle to continuously vary on the surface of the crystal analyser as $\left[z_{i} / R \sin \left(\Theta_{\mathrm{B}}-\alpha\right)\right]^{2} /$ $2 \tan \left(\Theta_{\mathrm{B}}-\alpha\right)$ (Suortti et al., 1999), where $z_{i}$ is the coordinate along the dispersive direction. The actual Johann aberration contribution to energy resolution is obtained from the histogram of the corresponding energy variations. For a crystal analyser with a circular mask of radius $A$ the resulting energy distribution is shown in Fig. 4 to be rather sharp, but with a long asymmetric tail. In this case, an estimate of the Johann aberration is given by

$$
\frac{\Delta \omega_{\mathrm{J}}}{\omega_{\mathrm{o}}}=\frac{\Delta \Theta_{\mathrm{J}}}{\tan \Theta_{\mathrm{B}}},
$$

where $\Delta \Theta_{\mathrm{J}}=\left[A / R \sin \left(\Theta_{\mathrm{B}}-\alpha\right)\right]^{2} / 8 \tan \left(\Theta_{\mathrm{B}}-\alpha\right)$ is the weighted average of the Bragg angle variation across the crystal analyser surface (inset of Fig. 4). The Johann aberration contribution is negligible when working close to backscattering geometry. In addition, it can be eliminated either by choosing $\alpha$ such that $\Theta_{\mathrm{B}}-\alpha=90^{\circ}$ or by using Johansson crystal analysers (Johansson, 1933). In the latter case, the reflecting lattice planes are bent to twice the Rowland circle radius, while the crystal analyser surface has the curvature of the Rowland circle. Their fabrication is, however, more complicated and Johansson spherical crystal analysers are not widely utilized yet. Otherwise, the Johann aberration can be minimized by limiting the active surface of the crystal analyser in the dispersive direction at the expense of a reduced solid angle.

2.2.3. Pixel-size contribution. When diced crystal analysers are used, the system becomes partially dispersive and photons with different energies are separated in angle/space. The image on the surface of the detector has the dimension of twice the cube size and features an energy gradient along the detector's vertical coordinate (Huotari et al., 2006). The 
energy resolution, then, depends on how finely this distribution of photon energies is sampled on the detector surface and a pixel size contribution is introduced,

$$
\frac{\Delta \omega_{\mathrm{p}}}{\omega_{\mathrm{o}}}=\frac{\Delta \Theta_{\mathrm{p}}}{\tan \Theta_{\mathrm{B}}},
$$

where $\Delta \Theta_{\mathrm{p}}=p / R\left[\sin \left(\Theta_{\mathrm{B}}+\alpha\right)+\sin \left(\Theta_{\mathrm{B}}-\alpha\right)\right]$, with $p$ the detector pixel size. When bent crystal analysers are used, instead, no exploitable energy gradient is produced on the detector surface. However, the spatial resolution of the detector can be used to enhance the energy resolution of a bent crystal analyser when it operates out-of-focus, as explained by Honkanen et al. (2014b).

2.2.4. Source-size contributions. Finally, one has to consider the finite source size, which introduces a distribution of point-like sources for the spectrometer. The three spatial dimensions $s_{x}, s_{y}$ and $s_{z}$ are determined by the focal spot size at the sample position, the beam incidence angle on the sample surface and the X-ray probing depth. We then define $s_{x_{i}}, s_{y_{i}}$ and $s_{z_{i}}$, the projections of the source in each crystal analyser's local coordinate system (top of Fig. 2), with $s_{x_{i}}$ and $s_{z_{i}}$ lying in the crystal analyser dispersive plane and $s_{y_{i}}$ perpendicular to it. $s_{z_{i}}$ and $s_{y_{i}}$ induce a Bragg angle variation, which is first order in $s_{z_{i}}, \Delta \Theta_{z_{i}}=s_{z_{i}} / R \sin \left(\Theta_{\mathrm{B}}-\alpha\right)$, and second order in $s_{y_{i}}, \Delta \Theta_{y_{i}}=$ $\left[s_{y_{\mathrm{i}}} / R \sin \left(\Theta_{\mathrm{B}}-\alpha\right)\right]^{2} \tan \Theta_{\mathrm{B}}$. Accordingly, the energy resolution is broadened by

$$
\frac{\Delta \omega_{y_{i}\left(z_{i}\right)}}{\omega_{\mathrm{o}}}=\frac{\Delta \Theta_{y_{i}\left(z_{i}\right)}}{\tan \Theta_{\mathrm{B}}}
$$

Finally, $s_{x_{i}}$ introduces a little off-Rowland contribution, which is typically negligible. In order to minimize source size contributions, the X-ray spot size on the sample has to be made as small as possible. At ID20, the source size contribution is negligible for most of the applications.

In the case of our multiple-element RIXS spectrometer, these contributions are almost identical for all the crystal analysers and so is their energy resolution. As an example, we consider the specific case of Ir $L_{3}$-edge RIXS experiments $\left(\omega_{\mathrm{o}}=11.215 \mathrm{keV}\right)$ employing five $R=2 \mathrm{~m} \mathrm{Si}(844)$ diced crystal analysers $\left(\Theta_{\mathrm{B}}=85.7^{\circ}\right)$, for which the various contributions to the energy resolution are $\Delta \omega_{\mathrm{a}}=14.7, \Delta \omega_{\mathrm{J}}=7.1$ (for a mask radius $A=30 \mathrm{~mm}), \Delta \omega_{\mathrm{p}}=11.5$ and $\Delta \omega_{z}=4.2 \mathrm{meV}$. The calculated overall energy resolution, including the incident photon energy bandwidth $\Delta \omega_{\mathrm{i}}=14.7 \mathrm{meV}$ determined by a $\mathrm{Si}(844)$ backscattering channel-cut, is $25.2 \mathrm{meV}$. Fig. 3(a) shows the foci of the five crystal analysers, which can be distinguished with the use of a two-dimensional detector. The displacement of the foci on the detector as a function of the incident photon energy is shown in the inset of Fig. 5. The slope of $4.7 \mu \mathrm{m} \mathrm{meV}^{-1}$ is related to the pixel size contribution to the energy resolution, $p / 4.7=11.7 \mathrm{meV}$ pixel $^{-1}$, in agreement with the estimate of $\Delta \omega_{\mathrm{p}}$ given above. The measured elastic lines are shown in Fig. 5 to be $25 \pm 3 \mathrm{meV}$ wide, again in line with the estimate above.

In order to optimize the energy resolution at all scattered photon energies, ID20 is equipped with a collection of crystal analysers (and post-monochromators). For convenience, we
Table 2

Calculated incident $\left(\Delta \omega_{\mathrm{i}}\right)$, crystal analyser $\left(\Delta \omega_{\mathrm{a}}\right)$, Johann aberration $\left(\Delta \omega_{\mathrm{J}}\right)$, pixel-size $\left(\Delta \omega_{\mathrm{p}}\right)$ and source-size $\left(\Delta \omega_{\mathrm{s}}\right)$ contributions to the spectrometer $\left(\Delta \omega_{\mathrm{o}}\right)$ energy resolution at selected absorption edges.

The overall calculated $\left(\Delta \omega_{\mathrm{t}}\right)$ and measured $\left(\Delta \omega_{\mathrm{m}}\right)$ energy resolutions are also reported. For $\Delta \omega_{\mathrm{i}}$ we considered the energy resolution provided by the asymmetric $\operatorname{Si}(n n n)$ four-bounce monochromator for the Ni $K$ - $(n=3)$ and Ta $L_{3}$-edges $(n=4)$, the asymmetric $\mathrm{Si}(440)$ four-bounce monochromator for the Pt $L_{3}$-edge, the $\mathrm{Si}(664)$ and $\mathrm{Si}(844)$ backscattering channel cuts for the Os and Ir $L_{3}$-edges, respectively. The radius of the crystal analyser mask is set to $A=$ $30 \mathrm{~mm}$ in all cases, except for the Ni $K$-edge, where $A=15 \mathrm{~mm}$.

\begin{tabular}{llllll}
\hline & Ni $K$ & Ta $L_{3}$ & Os $L_{3}$ & Ir $L_{3}$ & Pt $L_{3}$ \\
\hline$\omega_{\mathrm{i}}(\mathrm{keV})$ & 8.333 & 9.881 & 10.871 & 11.215 & 11.564 \\
Reflection & $\mathrm{Si}(551)$ & $\mathrm{Si}(660)$ & $\mathrm{Si}(664)$ & $\mathrm{Si}(844)$ & $\mathrm{Si}(771)$ \\
$\Theta_{\mathrm{B}}\left({ }^{\circ}\right)$ & 78.0 & 78.6 & 80.1 & 85.7 & 79.2 \\
$R(\mathrm{~m})$ & 1 & 2 & 2 & 2 & 1 \\
$\Delta \omega_{\mathrm{i}}(\mathrm{meV})$ & 55.9 & 40.2 & 17.2 & 14.7 & 80.0 \\
$\Delta \omega_{\mathrm{a}}(\mathrm{meV})$ & 26.5 & 23.4 & 17.2 & 14.7 & 10.1 \\
$\Delta \omega_{\mathrm{J}}(\mathrm{meV})$ & 11.0 & 11.8 & 9.7 & 1.8 & 49.6 \\
$\Delta \omega_{\mathrm{p}}(\mathrm{meV})$ & 49.7 & 28.0 & 26.6 & 11.5 & 62.0 \\
$\Delta \omega_{\mathrm{s}}(\mathrm{meV})$ & 18.1 & 10.2 & 9.7 & 4.2 & 22.6 \\
$\Delta \omega_{\mathrm{o}}(\mathrm{meV})$ & 60.2 & 39.6 & 34.5 & 19.3 & 86.6 \\
$\Delta \omega_{\mathrm{t}}(\mathrm{meV})$ & 82.2 & 56.4 & 38.5 & 24.3 & 117.9 \\
$\Delta \omega_{\mathrm{m}}(\mathrm{meV})$ & $93 \pm 5$ & $90 \pm 5$ & $52 \pm 3$ & $25 \pm 2$ & $216 \pm 12$ \\
& & & & &
\end{tabular}

report in Table 2 the calculated and measured energy resolutions at a few selected absorption edges.

\section{Energy calibration and stability}

With the setup for Ir $L_{3}$-edge RIXS, we checked the reliability on the energy scale of the RIXS spectrometer by measuring the well known dispersion of longitudinal acoustic phonons in diamond along the $(H, 0,0)$ direction. This is shown in Fig. 6 (open blue circles) to overlap with literature data (red line) (Warren et al., 1967). Finally, we also tested the stability of the energy scale in the long term; the inset of Fig. 6 shows the change in the phonon energy at $(3,0,0)$ momentum transfer over several hours. A small drift is observed, which can be

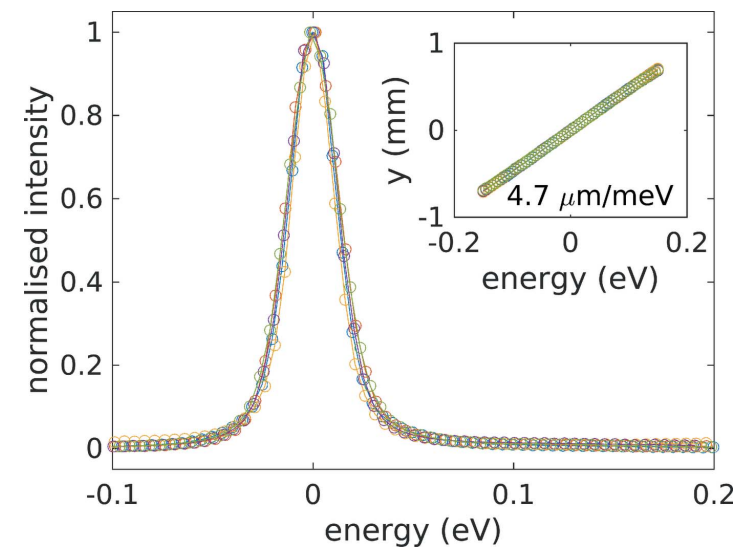

Figure 5

Measured elastic lines at $11.215 \mathrm{keV}$ employing five $R=2 \mathrm{~m} \mathrm{Si}(844)$ diced crystal analysers and a $\mathrm{Si}(844)$ backscattering channel-cut. The width of the curves is $25 \pm 3 \mathrm{meV}$. In the inset we track the displacement of the foci on the detector as a function of the incident photon energy. The slope of $4.7 \mu \mathrm{meV}^{-1}$ is related to the pixel size contribution to the energy resolution, $p / 4.7=11.7 \mathrm{meV}$ pixel $^{-1}$, in agreement with the estimate of $\Delta \omega_{\mathrm{p}}$ given in the text. 


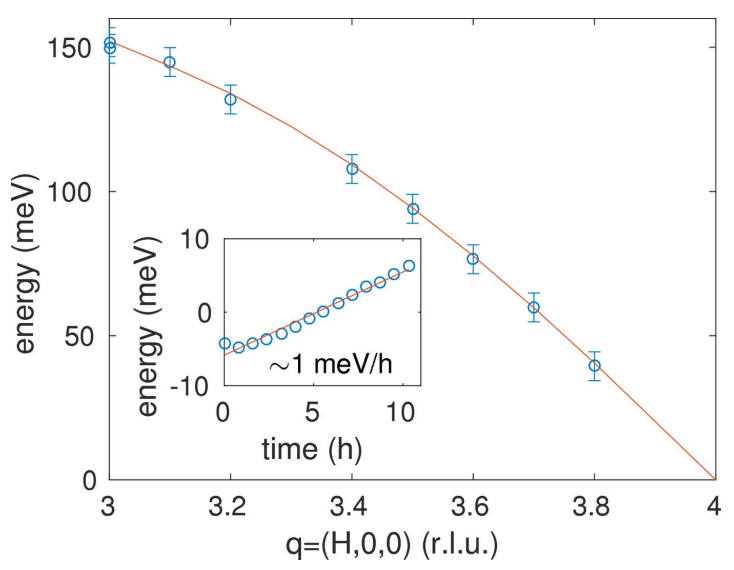

Figure 6

Phonon dispersion in diamond along the $(H, 0,0)$ direction measured with $\mathrm{Si}(844)$ crystal analysers $(R=2 \mathrm{~m})$ at $11.215 \mathrm{keV}$ and $25 \mathrm{meV}$ energy resolution (open blue circles), compared with literature data (red line). The inset shows the stability of the relative phonon energy versus time. A small drift of $\sim 1 \mathrm{meV} \mathrm{h}^{-1}$ is observed.

attributed to heat-load effects of the beamline optics and/or temperature changes in the experimental hutch. Indeed, heatload effects of the beamline optics cause a small change in the energy of the incident photons, as well as in the beam position on the sample. Note that a height change of the beam by $1 \mu \mathrm{m}$ corresponds to approximately $1 \mathrm{meV}$ in this case. Also, a temperature change of $0.1 \mathrm{~K}$ induces a $3 \mathrm{meV}$ shift in the scattered photon energy, given the linear thermal expansion coefficient of $\mathrm{Si}$ at room temperature of $2.6 \times 10^{-6} \mathrm{~K}^{-1}$. For this reason the temperature of the experimental hutch is stabilized within better than $0.5 \mathrm{~K}$ over $24 \mathrm{~h}$. Considering the effects above, an (in)stability of $\sim 1 \mathrm{meV} \mathrm{h}^{-1}$ over several hours is remarkably good, given that these measurements were performed in the 16-bunch mode of ESRF, which has the largest ring current changes associated with storage-ring refills out of all the current-filling modes practical for RIXS at ESRF. It should be mentioned, however, that experiments are performed for operation modes of the storage ring with smaller relative changes of the ring current upon refilling and energy drifts are usually negligible. Moreover, the future implementation of top-up at the ESRF is expected to mitigate these undesired effects related to heat-load variations for all operation modes of the storage ring.

\subsection{Scattering geometry}

Beside energy loss, momentum transfer is another important observable in RIXS experiments. It is defined as $\mathbf{q}=$ $\mathbf{k}_{\mathrm{o}}-\mathbf{k}_{\mathrm{i}}$ and it is varied by changing the scattering geometry, i.e. by tuning $\mathbf{k}_{\mathrm{o}}$, while keeping $\mathbf{k}_{\mathrm{i}}$ fixed. The RIXS spectrometer on beamline ID20 is capable of rotating the spectrometer arm in both the horizontal and vertical directions: as shown in Figs. $7(a)-7(d)$, the $\gamma_{0}$ rotation around the $z$ axis at the bottom of the spectrometer carries the $\delta_{0}$ rotation around the $y$ axis, thus allowing different scattering geometries.

In addition to providing full flexibility in the choice of the momentum transfer, the RIXS spectrometer also offers the possibility to study effects related to changes in the incident photon polarization. Indeed, the photon polarization in the horizontal laboratory plane corresponds to $\pi(\sigma)$ incident photon polarization when scattering in the horizontal (vertical) scattering plane. This way polarization effects can partially be investigated, despite the polarization of the scattered photons not being analysed. However, in order to minimize the source size contribution to the energy resolution, the scattering plane and the dispersive plane of the crystal analysers should be orthogonal. For this reason the RIXS chamber can be rotated around the source-to-middle crystal analyser axis, as shown in Figs. 7(b) and 7(c).

\section{Sample goniometer and momentum transfer}

A typical application of the RIXS spectrometer is the study of the momentum dependence of electronic and magnetic excitations in single crystals that need to be carefully aligned and oriented in order to reach specific points in reciprocal space. The sample stage therefore features three rotational and three translational degrees of freedom. As shown in Fig. 8, the translation stages are mounted on top of the rotation stages. The $\omega$ rotation around the $z$ axis at the bottom carries, in order, the $\chi$ and the $\varphi$ rotations, around the $x$ and $y$ axes, respectively. The sphere of confusion of the circles is less than (a)

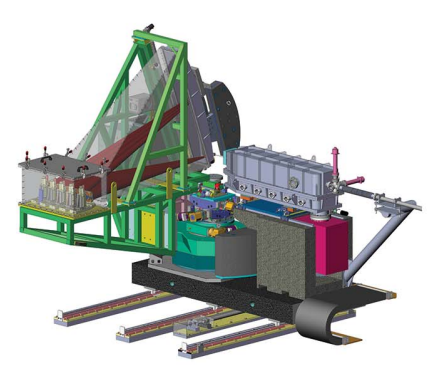

(b)

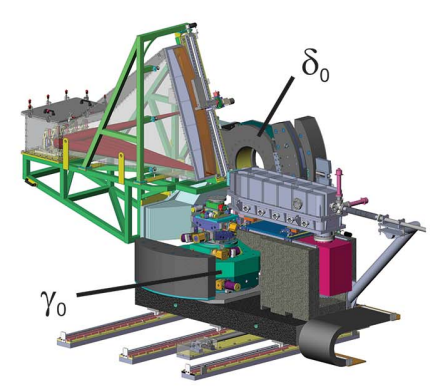

(c)

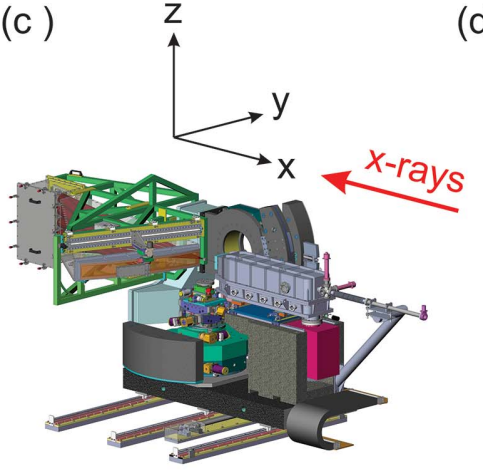

(d)

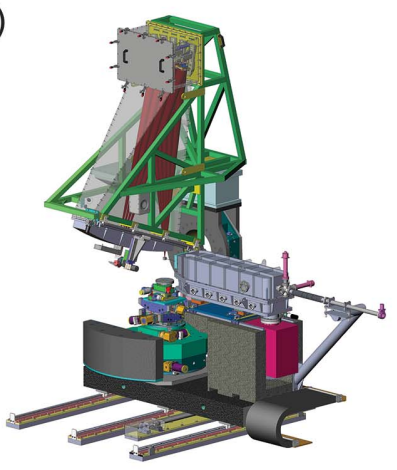

RIXS spectrometer equipped with the $R=2 \mathrm{~m}$ crystal analysers chamber in various scattering geometries for $\gamma_{0}=90^{\circ}$ and $\delta_{0}=0(a), \gamma_{0}=0$ and $\delta_{0}=0(b)$ and $(c), \gamma_{0}=0$ and $\delta_{0}=90^{\circ}(d)$. In $(b)$ the RIXS chamber is in the position for scattering in the horizontal plane, while in $(c)$ it is rotated around the source-to-middle crystal analyser axis by $90^{\circ}$ for scattering in the vertical plane. All combinations of these geometries are also possible. 

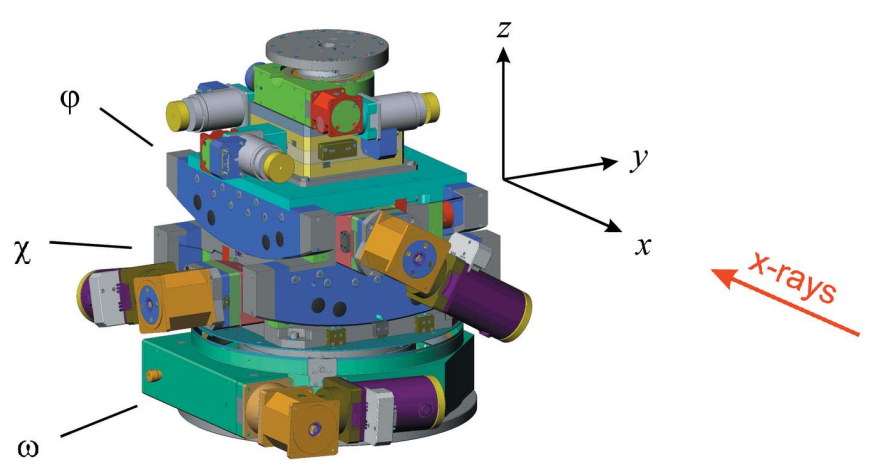

Figure 8

Sample tower of the RIXS spectrometer. Translation stages along the three orthogonal directions are mounted on top of the rotation stages. The $\omega$ rotation around the $z$ axis at the bottom carries, in order, the $\chi$ and the $\varphi$ rotations, around the $x$ and $y$ axes, respectively.

$50 \mu \mathrm{m}$. Details of the rotation and translation stages are given in Table 3.

Given the rotation of the spectrometer arm $\gamma_{0}$ and $\delta_{0}$ for the crystal analyser $i=0$, we obtain the angles

$$
\begin{aligned}
& \gamma_{i}=\gamma_{0}+\arctan \left(A_{i, y} / A_{i, x}\right), \\
& \delta_{i}=\delta_{0}+\arctan \left(A_{i, z} / A_{i, x}\right),
\end{aligned}
$$

where $A_{i, x}, A_{i, y}$ and $A_{i, z}$ are the positions of the $i$ th crystal analyser (see Appendix $A$ ). With the convention used in Fig. 7, the momentum transfer in the laboratory reference frame is given by

$$
\mathbf{q}_{i}^{2 \theta}=2 \pi\left(\frac{\hat{\mathbf{x}}}{\lambda_{\mathrm{i}}}-\frac{\mathcal{T}_{i}^{2 \theta} \hat{\mathbf{x}}}{\lambda_{\mathrm{o}}}\right),
$$

where $\mathcal{T}_{i}^{2 \theta}=\mathcal{R}_{\hat{\mathbf{z}}}\left(\gamma_{i}\right) \mathcal{R}_{\hat{\mathbf{y}}}\left(\delta_{i}\right)$ are the transformation matrices describing the rotation of the $2 \theta$-scattering arm. One then needs to project $\mathbf{q}_{i}^{2 \theta}$ onto the sample reciprocal space via

$$
\mathbf{q}_{i}^{h k l}=\left(\mathcal{T}^{\omega \chi \varphi} \mathcal{U B}\right)^{-1} \mathbf{q}_{i}^{2 \theta},
$$

where $\mathcal{T}^{\omega \chi \varphi}, \mathcal{U}$ and $\mathcal{B}$ are transformation matrices: $\mathcal{T}^{\omega \chi \varphi}=$ $\mathcal{R}_{\hat{\mathbf{z}}}(\omega) \mathcal{R}_{\hat{\mathbf{x}}}(\chi) \mathcal{R}_{\hat{\mathbf{y}}}(\varphi)$ describes the sample rotations; $\mathcal{U}$ links the laboratory and the sample reference frames. It depends on how the sample is mounted on the sample stage and is found through the sample alignment procedure; finally, $\mathcal{B}$ links the sample real space to its reciprocal space reference frame and so it contains the dependence on the lattice parameters. Knowledge of these matrices allows one to easily obtain the momentum transfer associated with the various crystal analysers. Similarly, they can be used to estimate the momentum resolution. Details of the formalism are given in Appendix $B$.

\section{Conclusions and outlook}

We have presented a multiple-element RIXS spectrometer installed at beamline ID20 at the ESRF. It has been conceived in order to maximize the collection solid angle, optimize the energy resolution and allow a flexible choice of the scattering
Table 3

Details of the sample tower and scattering arm rotation and translation stages.

\begin{tabular}{llll}
\hline Name & Description & Stroke & Resolution \\
\hline & Spectrometer translation & $0-120 \mathrm{~mm}$ & $2.4 \mu \mathrm{m}$ \\
$\gamma_{0}$ & Arm rotation & $-35-150^{\circ}$ & $15 \mu \mathrm{rad}$ \\
$\delta_{0}$ & Arm rotation & $-15-150^{\circ}$ & $15 \mu \mathrm{rad}$ \\
$x$ & Sample translation & $\pm 15 \mathrm{~mm}$ & $0.5 \mu \mathrm{m}$ \\
$y$ & Sample translation & $\pm 15 \mathrm{~mm}$ & $0.5 \mu \mathrm{m}$ \\
$z$ & Sample translation & $\pm 20 \mathrm{~mm}$ & $0.02 \mu \mathrm{m}$ \\
$\omega$ & Sample rotation & $\pm 180^{\circ}$ & $10 \mu \mathrm{rad}$ \\
$\chi$ & Sample rotation & $\pm 7^{\circ}$ & Non-linear \\
$\varphi$ & Sample rotation & $\pm 7^{\circ}$ & Non-linear \\
\hline
\end{tabular}

geometry, for momentum and/or polarization resolved studies of the electronic structure of materials. It is mainly dedicated to the investigation of low-energy electronic excitation, but it can be exploited as well to the study of higher energy excitations or emission lines.

Future developments may include improvements of the energy resolution via the use of crystal analysers of different materials, such as lithium niobate $\left(\mathrm{LiNbO}_{3}\right)$, sapphire $\left(\mathrm{Al}_{2} \mathrm{O}_{3}\right)$ and $\alpha$-quartz $\left(\mathrm{SiO}_{2}\right)$. These materials have crystal structures of lower symmetry and thus offer more possible reflections than $\mathrm{Si}$ or Ge, with numerous choices of intrinsic resolution and throughput (Gog et al., 2013; Ketenoglu et al., 2015; Yavaş et al., 2017). Another option is to adopt different optical schemes than that of a conventional Rowland spectrometer, such as for example those based on the CDW design and its variants introduced by Shvyd'ko et al. (2006, 2011, 2014). In addition, future developments may comprise a smaller focal spot size, which will facilitate high-pressure experiments and highenergy-resolution applications. Also beneficial to energy resolution could be the advent of two-dimensional detectors with higher spatial resolution.

\section{APPENDIX $A$ \\ Multiple Rowland circles}

We consider here the case of five identical crystal analysers and explain how the position (linear and angular) of the various crystal analysers and detector are determined as a function of the Bragg angle $\Theta_{B}$. The position of the source, crystal analyser and its focus on the surface of the detector is described by the vectors $\mathbf{S}_{i}, \mathbf{A}_{i}$ and $\mathbf{D}_{i}$, respectively, where $i=$ $0, \pm 1, \pm 2$ labels the five crystal analysers. Similarly, the centre of the Rowland circle is located at $\mathbf{R}_{i}$. By introducing a reference system with the origin at the source and oriented as in Fig. 9(a), the one-crystal analyser case $(i=0)$ is described by $S_{0, x}=S_{0, y}=S_{0, z}=0$ for the source, $A_{0, x}=R \sin \left(\Theta_{\mathrm{B}}-\alpha\right), A_{0, y}=$ $A_{0, z}=0$ for the crystal analyser, $D_{0, x}=R \cos \left(\Theta_{\mathrm{B}}+\alpha\right) \sin 2 \Theta_{\mathrm{B}}$, $D_{0, y}=0, D_{0, z}=R \sin \left(\Theta_{\mathrm{B}}+\alpha\right) \sin 2 \Theta_{\mathrm{B}}$ for the detector and $R_{0, x}=R \sin \left(\Theta_{\mathrm{B}}-\alpha\right) / 2, R_{0, y}=0, R_{0, z}=R \cos \left(\Theta_{\mathrm{B}}-\alpha\right) / 2$ for the centre of the Rowland circle, where $\alpha$ allows for an asymmetric reflection.

The position of the generic $i$ th crystal analyser should be calculated taking into account that (i) the Rowland circles intersect at the common source position and that choices in 
(a)

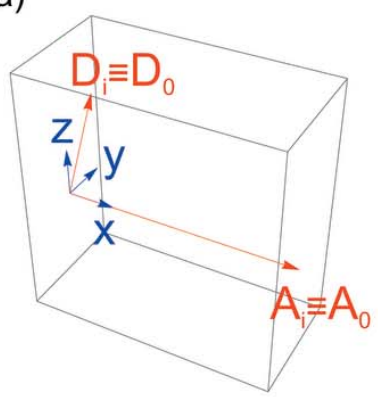

(b)

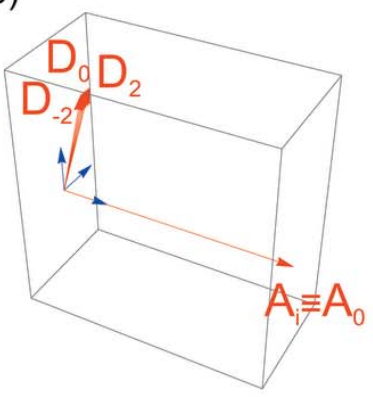

(c)

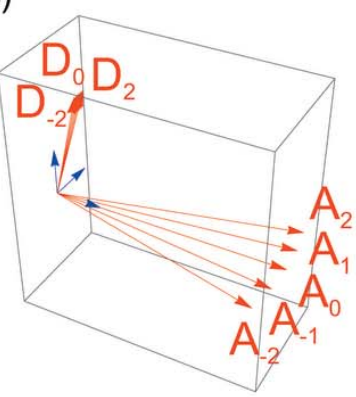

(d)

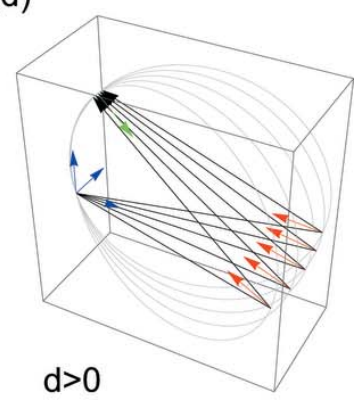

(e)

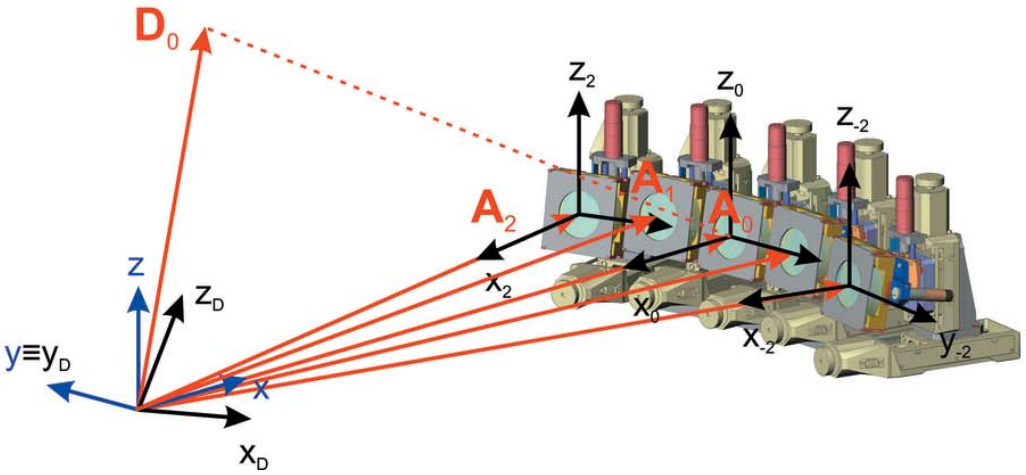

(f)

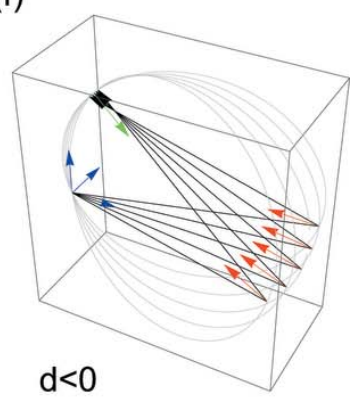

Figure 9

The position of crystal analysers and detector is determined starting from the one crystal analyser case $(a)$. The Cartesian reference system is also shown. Two successive rotations are applied as explained in the text; their effect can be seen in panels $(b)$ and $(c)$, respectively. Panels $(d)$ and $(f)$ show the crystal analysers and detector position, as well as their surface normals in the two configurations, $d>0$ and $d<0$. Panel $(e)$ shows the crystal analysers assembly and the crystals analysers and detector local coordinate systems.

the mechanical implementation of the multiple crystal analysers spectrometer impose that (ii) the foci of the various crystal analysers should lie on, but not necessarily coincide with, the detector surface; (iii) the crystal analysers move on a translation stage directed towards the source. These stages are fixed on the spectrometer assembly and form an angle $\beta_{i}$ with respect to the central analyser translation.

For the generic $i$ th crystal analyser case, constraint (i) imposes that $\mathbf{S}_{i}=\mathbf{S}_{0}=0$. $\mathbf{A}_{i}$ and $\mathbf{D}_{i}$ are then determined by applying two consecutive rotations to the reference Rowland circle $(i=0)$ as explained in the following. Initially $\mathbf{A}_{i}=\mathbf{A}_{0}$ and $\mathbf{D}_{i}=\mathbf{D}_{0}$. The first rotation $\mathcal{R}_{\mathbf{x}}\left(\psi_{i}\right)$ is around the $\hat{\mathbf{x}}$ axis by an angle $\psi_{i}$, and affects the position of the focus on the detector,

$$
\mathbf{D}_{i}=\mathcal{R}_{\hat{\mathbf{x}}}\left(\psi_{i}\right) \mathbf{D}_{0}
$$

while leaving the position of the crystal analyser unchanged, $\mathbf{A}_{i}=\mathcal{R}_{\hat{\mathbf{x}}}\left(\psi_{i}\right) \mathbf{A}_{0}=\mathbf{A}_{0}$, as is shown in Fig. 9(b). Constraint (ii) requires that

$$
\begin{gathered}
D_{i, x}=D_{0, x}, \\
D_{i, y}=d_{i},
\end{gathered}
$$

where $d_{i}$ is the distance between the foci on the surface of the detector from its centre. Typically, $d_{i}=i d$, with $|d| \leq 14 \mathrm{~mm}$, the lateral size of the individual chips. For $d=0$ all the crystal analysers focus at the centre of the detector, while for $d \neq 0$ each individual contribution can be separated. It is easy to see that equation (12) is fulfilled for $\sin \psi_{i}=-i d / D_{0, z}$, while equation (11) is naturally satisfied as $\mathcal{R}_{\hat{\mathbf{x}}}\left(\psi_{i}\right)$ does not affect the $D_{i, x}$ components.

The final crystal analyser position is obtained by applying a second rotation $\mathcal{R}_{\mathbf{D}_{i}}\left(\eta_{i}\right)$ around $\mathbf{D}_{i}$ by an angle $\eta_{i}$,

$$
\mathbf{A}_{i}=\mathcal{R}_{\mathbf{D}_{i}}\left(\eta_{i}\right) \mathbf{A}_{0} .
$$

Obviously, this rotation does not alter the position of the focus on the detector, $\mathcal{R}_{\mathbf{D}_{\mathbf{i}}}\left(\eta_{i}\right) \mathbf{D}_{i}=\mathbf{D}_{i}$, as is shown in Fig. $9(c)$. Constraint (iii) imposes that

$$
A_{i, y}=A_{i, x} \tan \beta_{i},
$$

where $\beta_{i}$ is the angle between the translation of the $i$ th crystal analyser and the central one. By mechanical construction, $\beta_{i}=i \beta$, where $\beta=7^{\circ}$ and $3.5^{\circ}$ for the $R=1 \mathrm{~m}$ and $2 \mathrm{~m}$ case, respectively. Equation (14) is fulfilled for $\eta_{i}$ that satisfies

$$
\begin{aligned}
& D_{0, z}\left[D_{0, z} \tan \beta_{i}-D_{0, x} \sin \psi_{i}\right] \cos \eta_{i}= \\
& \quad D_{0, z}\left|\mathbf{D}_{0}\right| \cos \psi_{i} \sin \eta_{i}-D_{0, x}^{2} \tan \beta_{i}-D_{0, x} D_{0, z} \sin \psi_{i} .
\end{aligned}
$$

Finally, the centre of the Rowland circles is given by

$$
\mathbf{R}_{i}=\mathcal{R}_{\mathbf{D}_{i}}\left(\eta_{i}\right) \mathcal{R}_{\hat{\mathbf{x}}}\left(\psi_{i}\right) \mathbf{R}_{0},
$$

and their orientation is such to intersect the corresponding source, crystal analyser and detector, as shown in Fig. $9(d)$. In addition, in order to correctly orient the crystal analysers and the detector, it is useful to consider the normal to the crystal analysers surface,

$$
\mathbf{n}_{i}^{\mathrm{a}}=\mathcal{R}_{\mathbf{D}_{i}}\left(\eta_{i}\right) \mathcal{R}_{\hat{\mathbf{x}}}\left(\psi_{i}\right) \mathbf{n}_{0}^{\mathrm{a}},
$$


where $\mathbf{n}_{0}^{\mathrm{a}}=\left(\mathbf{D}_{0}-2 \mathbf{A}_{0}\right) /\left|\mathbf{D}_{0}-2 \mathbf{A}_{0}\right|$, and the normal to the detector surface,

$$
\mathbf{n}_{i}^{\mathrm{D}}=\mathcal{R}_{\mathbf{D}_{i}}\left(\eta_{i}\right) \mathcal{R}_{\hat{\mathbf{x}}}\left(\psi_{i}\right) \mathbf{n}_{0}^{\mathrm{D}},
$$

where $\mathbf{n}_{0}^{\mathrm{D}}=\left(\mathbf{R}_{0}-\mathbf{D}_{0}\right) /\left|\mathbf{R}_{0}-\mathbf{D}_{0}\right|$. These are also shown in Fig. $9(d)$. Ultimately, a variation of the spectrometer energy involves two rotations $\left(\theta_{i}, \chi_{i}\right)$ and two translations $\left(x_{i}, z_{i}\right)$ of the crystal analysers, and one rotation $\left(\theta_{\mathrm{D}}\right)$ and two translations $\left(x_{\mathrm{D}}, z_{\mathrm{D}}\right)$ of the detector, which are calculated from $\mathbf{n}_{i}^{\mathrm{a}}$ and $\mathbf{n}_{i}^{\mathrm{D}}$, respectively. Finally, Figs. $9(d)$ and $9(f)$ show two spectrometer configurations depending on the sign of $d$ : for $d>0$ the foci of the crystal analysers do not cross on the detector, while they do for $d<0$.

\section{APPENDIX $B$}

\section{$\mathcal{U}, \mathcal{B}$ matrices, momentum transfer and momentum resolution}

As mentioned above, the calculation of the momentum transfer in the sample reciprocal space requires knowledge of the $\mathcal{U}$ and $\mathcal{B}$ matrices. The former is related to how the sample is mounted in the laboratory reference frame, namely it defines the sample orientation before any rotation is applied. The latter defines a Cartesian reference frame in the sample reciprocal space and therefore it contains the dependence on the lattice constants. Following the convention of Busing \& Levy (1967), the definition of $\mathcal{B}$ is reported here for convenience,

$$
\mathcal{B}=\left(\begin{array}{ccc}
a^{*} & b^{*} \cos \gamma^{*} & c * \cos \beta^{*} \\
0 & b^{*} \sin \gamma^{*} & -c^{*} \sin \beta^{*} \cos \alpha \\
0 & 0 & 1 / c
\end{array}\right),
$$

with the usual definitions of the primitive and reciprocal lattice parameters.

The momentum transfer given by equation (9) depends on the $\mathcal{U}$ matrix, which needs to be determined prior to the experiment. The typical procedure is to find two non-collinear Bragg reflections and use them to derive the sample orientation in the laboratory reference frame. Ideally, the $\mathcal{U}$ matrix satisfies the equations

$$
\begin{aligned}
& \mathbf{q}_{1}^{h k l}=\left(\mathcal{T}_{1}{ }^{\omega \chi \varphi} \mathcal{U B}\right)^{-1} \mathbf{q}_{1}^{2 \theta}, \\
& \mathbf{q}_{2}^{h k l}=\left(\mathcal{T}_{2}{ }^{\omega \chi \varphi} \mathcal{U B}\right)^{-1} \mathbf{q}_{2}^{2 \theta}
\end{aligned}
$$

simultaneously, where $\mathbf{q}_{1}^{h k l}\left(\mathbf{q}_{2}^{h k l}\right)$ is the first (second) Bragg reflection expressed in the sample reciprocal space, while $\mathcal{T}_{1}^{\omega \chi \varphi}\left(\mathcal{T}_{2}^{\omega \chi \varphi}\right)$ and $\mathbf{q}_{1}^{2 \theta}\left(\mathbf{q}_{2}^{2 \theta}\right)$ contain information on the scattering arm and sample angles for which the first (second) Bragg reflection is found. However, because of experimental errors in the angle measurements and/or uncertainties in the cell parameters, it is not in general possible to find an orthogonal matrix $\mathcal{U}$ which satisfies both conditions. In order to avoid this difficulty it is generally required that $\mathbf{q}_{1}^{h k l}$ is parallel to $\mathbf{q}_{1}^{2 \theta}$, and $\mathbf{q}_{2}^{h k l}$ is only constrained to lie in the plane of $\mathbf{q}_{1}^{2 \theta}$ and $\mathbf{q}_{2}^{2 \theta}$ (Busing \& Levy, 1967). By defining

$$
\begin{gathered}
\mathbf{t}_{1}=\mathcal{B} \mathbf{q}_{1}^{h k l}, \\
\mathbf{t}_{3}=\mathcal{B}\left(\mathbf{q}_{1}^{h k l} \times \mathbf{q}_{2}^{h k l}\right) . \\
\mathbf{t}_{2}=\mathbf{t}_{1} \times \mathbf{t}_{3},
\end{gathered}
$$

and

$$
\begin{gathered}
\mathbf{v}_{1}=\mathcal{T}_{1}^{\omega \chi \varphi-1} \mathbf{q}_{1}^{2 \theta}, \\
\mathbf{v}_{3}=\mathcal{T}_{1}^{\omega \chi \varphi-1} \mathbf{q}_{1}^{2 \theta} \times \mathcal{T}_{2}^{\omega \chi \varphi-1} \mathbf{q}_{2}^{2 \theta}, \\
\mathbf{v}_{2}=\mathbf{v}_{1} \times \mathbf{v}_{3},
\end{gathered}
$$

then the $\mathcal{U}$ matrix can be found as

$$
\mathcal{U}=\mathcal{V} \mathcal{T}^{-1}
$$

where $\mathcal{T}=\left(\mathbf{t}_{1} /\left|\mathbf{t}_{1}\right|, \mathbf{t}_{2} /\left|\mathbf{t}_{2}\right|, \mathbf{t}_{3} /\left|\mathbf{t}_{3}\right|\right)$ and $\mathcal{V}=\left(\mathbf{v}_{1} /\left|\mathbf{v}_{1}\right|, \mathbf{v}_{2} /\left|\mathbf{v}_{2}\right|\right.$, $\left.\mathbf{v}_{3} /\left|\mathbf{v}_{3}\right|\right)$. If the $\mathcal{U}$ and $\mathcal{B}$ matrices are known, the sample $(\omega, \chi$ and $\varphi$ ) and the scattering arm $\left(\gamma_{i}\right.$ and $\left.\delta_{i}\right)$ angles can be calculated in order to reach a well defined momentum transfer $\mathbf{q}_{i}^{h k l}$.

Momentum resolution is related to uncertainties in the scattering arm angles $(\Delta \gamma$ and $\Delta \delta)$ and ultimately to the finite aperture $(A)$ of the crystal analysers by

$$
\Delta \mathbf{q}_{i}^{h k l} \simeq\left(\mathcal{T}^{\omega \chi \varphi} \mathcal{U B}\right)^{-1}\left(\frac{\partial \mathbf{q}_{i}^{2 \theta}}{\partial \gamma_{i}} \Delta \gamma+\frac{\partial \mathbf{q}_{i}^{2 \theta}}{\partial \delta_{i}} \Delta \delta\right),
$$

where $\Delta \gamma$ and $\Delta \delta$ are both of the order of $A /\left|\mathbf{A}_{i}\right|$ (for $A \ll$ $\left.\left|\mathbf{A}_{i}\right|\right)$. In the case of (quasi)elastic scattering, $\lambda_{\mathrm{o}} \simeq \lambda_{\mathrm{i}}=\lambda$ and the expression above simplifies to

$$
\Delta \mathbf{q}_{i}^{h k l} \simeq\left(\mathcal{T}^{\omega \chi \varphi} \mathcal{U B}\right)^{-1} \mathcal{T}^{2 \theta} \Delta \mathbf{q}_{i}^{2 \theta},
$$

where $\Delta \mathbf{q}_{i}^{2 \theta}=2 \pi\left(0,-\Delta \gamma \cos \delta_{i}, \Delta \delta\right) / \lambda$. As a typical example, we discuss the case of Ir $L_{3}$-edge RIXS of a $\operatorname{Sr}_{3} \operatorname{Ir}_{3} \mathrm{O}_{7}$ single crystal ideally oriented with the $(-1,-1,0)$ direction along the $x$ axis and the $(0,0,-1)$ direction along the $y$ axis when all sample rotations are set to zero. We consider the case for $\delta_{0}=$ $2 \omega=90^{\circ}$ and $\gamma_{0}=\chi=\varphi=0$, for which $\mathbf{q}_{0}^{h k l}=(0,0,26.7)$ r.l.u. Figs. $10(a), 10(c)$ and $10(e)$ show the momentum transfer spread across the surface of the crystal analyser $(R=2 \mathrm{~m})$ with a circular aperture of radius $A=30 \mathrm{~mm}$. The corresponding width of the momentum resolution function is shown in Figs. $10(b), 10(d)$ and $10(f)$ to be different in the various reciprocal lattice directions. Note that the shape of the crystal analyser mask could be varied in order to optimize the momentum transfer resolution in a specific direction depending on the experimental requirements. In any case, the finite momentum resolution of the spectrometer should be considered, in particular when interpreting REXS and RIXS results.

\section{Acknowledgements}

We kindly acknowledge the ESRF for financing the UPBL6 project as part of the ESRF Upgrade - Phase I. We also would like thank the ESRF for support during the conception, design, construction and commissioning of the spectrometer, specifically the TID and ISDD divisions. 
(a)

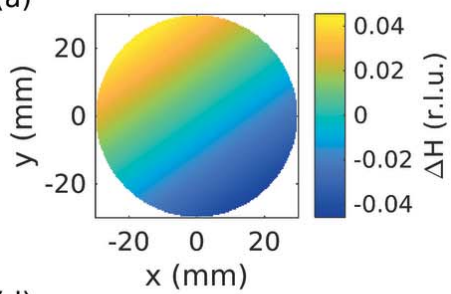

(d)

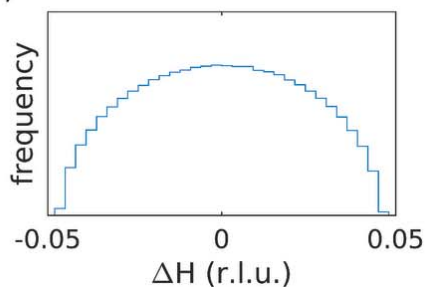

(b)

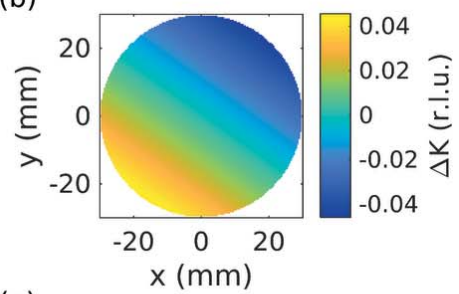

(e)

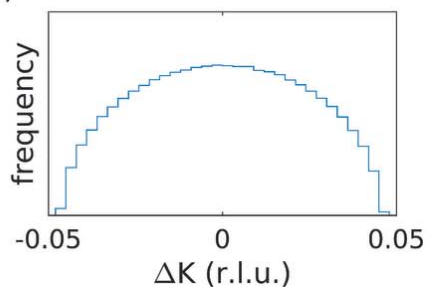

(c)

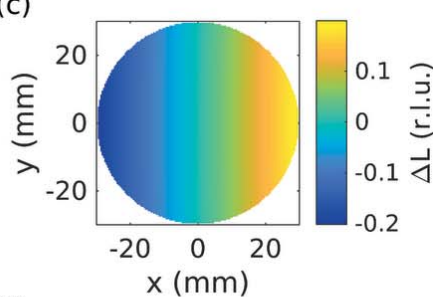

(f)

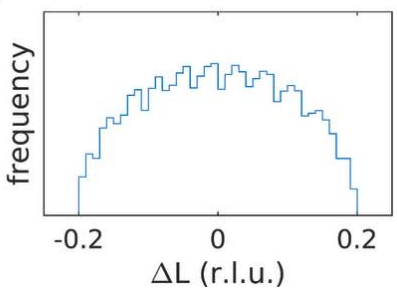

Figure 10

Momentum resolution in the case of $\operatorname{Ir} L_{3}$-edge RIXS of a $\operatorname{Sr}_{3} \operatorname{Ir}_{2} \mathrm{O}_{7}\left(a=b=3.896 \AA, c=20.88 \AA\right.$ and $\left.\alpha=\beta=\gamma=90^{\circ}\right)$ single crystal ideally oriented with the $(-1,-1,0)$ direction along the $x$ and the $(0,0,-1)$ direction along the $y$ axis when all sample rotations are set to zero. Sample and spectrometer are aligned such that $\mathbf{q}_{0}^{h k l}=(0,0,26.5)$ r.l.u., i.e. $\delta_{0}=2 \omega=90^{\circ}$ and $\gamma_{0}=\chi=\varphi=0$. Panels $(a),(c)$ and $(e)$ show the momentum transfer spread along the reciprocal lattice axes across the surface of a crystal analyser $(R=2 \mathrm{~m})$ with a circular aperture of radius $A=30 \mathrm{~mm}$. Panels $(b),(d)$ and $(f)$ show the corresponding momentum resolution functions.

\section{References}

Ament, L. J. P., Khaliullin, G. \& van den Brink, J. (2011b). Phys. Rev. $B, 84,020403$.

Ament, L. J. P., van Veenendaal, M., Devereaux, T. P., Hill, J. P. \& van den Brink, J. (2011a). Rev. Mod. Phys. 83, 705-767.

Ament, L. J. P., van Veenendaal, M. \& van den Brink, J. (2011c). Europhys. Lett. 95, 27008.

Bauer, M. (2014). Phys. Chem. Chem. Phys. 16, 13827-13837.

Benckiser, E., Fels, L., Ghiringhelli, G., Moretti Sala, M., Schmitt, T., Schlappa, J., Strocov, V. N., Mufti, N., Blake, G. R., Nugroho, A. A., Palstra, T. T. M., Haverkort, M. W., Wohlfeld, K. \& Grüninger, M. (2013). Phys. Rev. B, 88, 205115.

Braicovich, L., Minola, M., Dellea, G., Le Tacon, M., Moretti Sala, M., Morawe, C., Peffen, J.-C., Supruangnet, R., Yakhou, F., Ghiringhelli, G. \& Brookes, N. B. (2014). Rev. Sci. Instrum. 85, 115104.

Braicovich, L., van den Brink, J., Bisogni, V., Sala, M. M., Ament, L. J. P., Brookes, N. B., De Luca, G. M., Salluzzo, M., Schmitt, T., Strocov, V. N. \& Ghiringhelli, G. (2010). Phys. Rev. Lett. 104, 077002 .

Brink, J. van den \& van Veenendaal, M. (2012). Synchrotron Radiat. News, 25(4), 29-32.

Busing, W. R. \& Levy, H. A. (1967). Acta Cryst. 22, 457-464.

Calder, S., Kim, J. W., Taylor, A. E., Upton, M. H., Casa, D., Cao, G., Mandrus, D., Lumsden, M. D. \& Christianson, A. D. (2016). Phys. Rev. B, 94, 220407.

Cao, Y., Liu, X., Xu, W., Yin, W.-G., Meyers, D., Kim, J., Casa, D., Upton, M. H., Gog, T., Berlijn, T., Alvarez, G., Yuan, S., Terzic, J., Tranquada, J. M., Hill, J. P., Cao, G., Konik, R. M. \& Dean, M. P. M. (2017). Phys. Rev. B, 95, 121103.

Clancy, J. P., Gretarsson, H., Lee, E. K. H., Tian, D., Kim, J., Upton, M. H., Casa, D., Gog, T., Islam, Z., Jeon, B.-G., Kim, K. H., Desgreniers, S., Kim, Y. B., Julian, S. J. \& Kim, Y.-J. (2016). Phys. Rev. B, 94, 024408.

Cudazzo, P., Ruotsalainen, K. O., Sahle, C. J., Al-Zein, A., Berger, H., Navarro-Moratalla, E., Huotari, S., Gatti, M. \& Rubio, A. (2014). Phys. Rev. B, 90, 125125.

Dean, M. P. M., Dellea, G., Minola, M., Wilkins, S. B., Konik, R. M., Gu, G. D., Le Tacon, M., Brookes, N. B., Yakhou-Harris, F., Kummer, K., Hill, J. P., Braicovich, L. \& Ghiringhelli, G. (2013a). Phys. Rev. B, 88, 020403.
Dean, M. P. M., James, A. J. A., Springell, R. S., Liu, X., Monney, C., Zhou, K. J., Konik, R. M., Wen, J. S., Xu, Z. J., Gu, G. D., Strocov, V. N., Schmitt, T. \& Hill, J. P. (2013b). Phys. Rev. Lett. 110, 147001.

Dean, M. P. M., James, A. J. A., Walters, A. C., Bisogni, V., Jarrige, I., Hücker, M., Giannini, E., Fujita, M., Pelliciari, J., Huang, Y. B., Konik, R. M., Schmitt, T. \& Hill, J. P. (2014). Phys. Rev. B, 90, 220506.

Dean, M. P. M., Springell, R. S., Monney, C., Zhou, K. J., Pereiro, J., Božović, I., Dalla Piazza, B., Rønnow, H. M., Morenzoni, E., van den Brink, J., Schmitt, T. \& Hill, J. P. (2012). Nat. Mater. 11, 850-854.

Devereaux, T. P., Shvaika, A. M., Wu, K., Wohlfeld, K., Jia, C. J., Wang, Y., Moritz, B., Chaix, L., Lee, W.-S., Shen, Z.-X., Ghiringhelli, G. \& Braicovich, L. (2016). Phys. Rev. X, 6, 041019.

Donnerer, C., Rahn, M. C., Sala, M. M., Vale, J. G., Pincini, D., Strempfer, J., Krisch, M., Prabhakaran, D., Boothroyd, A. T. \& McMorrow, D. F. (2016). Phys. Rev. Lett. 117, 037201.

Duan, P., Gu, S., Cao, H., Li, J. \& Huang, Y. (2017). X-ray Spectrom. 46, 12-18.

Gao, X., Burns, C., Casa, D., Upton, M., Gog, T., Kim, J. \& Li, C. (2011). Rev. Sci. Instrum. 82, 113108.

Ghiringhelli, G. \& Braicovich, L. (2013). J. Electron Spectrosc. Relat. Phenom. 188, 26-31.

Ghiringhelli, G., Brookes, N. B., Annese, E., Berger, H., Dallera, C., Grioni, M., Perfetti, L., Tagliaferri, A. \& Braicovich, L. (2004). Phys. Rev. Lett. 92, 117406.

Ghiringhelli, G., Le Tacon, M., Minola, M., Blanco-Canosa, S., Mazzoli, C., Brookes, N. B., De Luca, G. M., Frano, A., Hawthorn, D. G., He, F., Loew, T., Moretti Sala, M., Peets, D. C., Salluzzo, M., Schierle, E., Sutarto, R., Sawatzky, G. A., Weschke, E., Keimer, B. \& Braicovich, L. (2012). Science, 337, 821-825.

Ghiringhelli, G., Piazzalunga, A., Dallera, C., Trezzi, G., Braicovich, L., Schmitt, T., Strocov, V. N., Betemps, R., Patthey, L., Wang, X. \& Grioni, M. (2006). Rev. Sci. Instrum. 77, 113108.

Glatzel, P. \& Bergmann, U. (2005). Coord. Chem. Rev. 249, 65-95.

Glatzel, P., de Groot, F. M. F. \& Bergmann, U. (2009). Synchrotron Radiat. News, 22, 12-16.

Gog, T., Casa, D. M., Said, A. H., Upton, M. H., Kim, J., Kuzmenko, I., Huang, X. \& Khachatryan, R. (2013). J. Synchrotron Rad. 20, 74-79. 
Gretarsson, H., Sung, N. H., Porras, J., Bertinshaw, J., Dietl, C., Bruin, J. A. N., Bangura, A. F., Kim, Y. K., Dinnebier, R., Kim, J., Al-Zein, A., Moretti Sala, M., Krisch, M., Le Tacon, M., Keimer, B. \& Kim, B. J. (2016). Phys. Rev. Lett. 117, 107001.

Guarise, M., Dalla Piazza, B., Berger, H., Giannini, E., Schmitt, T., Rønnow, H. M., Sawatzky, G. A., van den Brink, J., Altenfeld, D., Eremin, I. \& Grioni, M. (2014). Nat. Commun. 5, 5760.

Guarise, M., Dalla Piazza, B., Moretti Sala, M., Ghiringhelli, G., Braicovich, L., Berger, H., Hancock, J. N., van der Marel, D., Schmitt, T., Strocov, V. N., Ament, L. J. P., van den Brink, J., Lin, P., Xu, P., Rønnow, H. M. \& Grioni, M. (2010). Phys. Rev. Lett. 105, 157006.

Haverkort, M. W. (2010). Phys. Rev. Lett. 105, 167404.

Honkanen, A.-P., Verbeni, R., Simonelli, L., Moretti Sala, M., Al-Zein, A., Krisch, M., Monaco, G. \& Huotari, S. (2014b). J. Synchrotron Rad. 21, 762-767.

Honkanen, A.-P., Verbeni, R., Simonelli, L., Moretti Sala, M., Monaco, G. \& Huotari, S. (2014c). J. Synchrotron Rad. 21, 104-110.

Honkanen, A.-P., Verbeni, R., Simonelli, L., Moretti Sala, M., Monaco, G. \& Huotari, S. (2017). J. Synchrotron Rad. 24, 545-546.

Huotari, S., Albergamo, F., Vankó, G., Verbeni, R. \& Monaco, G. (2006). Rev. Sci. Instrum. 77, 053102.

Huotari, S., Pylkkänen, T., Verbeni, R., Monaco, G. \& Hämäläinen, K. (2011). Nat. Mater. 10, 489-493.

Huotari, S., Sahle, C. J., Henriquet, C., Al-Zein, A., Martel, K., Simonelli, L., Verbeni, R., Gonzalez, H., Lagier, M.-C., Ponchut, C., Moretti Sala, M., Krisch, M. \& Monaco, G. (2017). J. Synchrotron Rad. 24, 521-530.

Huotari, S., Vankó, Gy., Albergamo, F., Ponchut, C., Graafsma, H., Henriquet, C., Verbeni, R. \& Monaco, G. (2005). J. Synchrotron Rad. 12, 467-472.

Hwan Chun, S., Kim, J.-W., Kim, J., Zheng, H., Stoumpos, C. C., Malliakas, C. D., Mitchell, J. F., Mehlawat, K., Singh, Y., Choi, Y., Gog, T., Al-Zein, A., Sala, M. M., Krisch, M., Chaloupka, J., Jackeli, G., Khaliullin, G. \& Kim, B. J. (2015). Nat. Phys. 11, 462-466.

Igarashi, J. \& Nagao, T. (2012). Phys. Rev. B, 85, 064421.

Ishii, K., Fujita, M., Sasaki, T., Minola, M., Dellea, G., Mazzoli, C., Kummer, K., Ghiringhelli, G., Braicovich, L., Tohyama, T., Tsutsumi, K., Sato, K., Kajimoto, R., Ikeuchi, K., Yamada, K., Yoshida, M., Kurooka, M. \& Mizuki, J. (2014). Nat. Commun. 5, 3714.

Ishii, K., Ishihara, S., Murakami, Y., Ikeuchi, K., Kuzushita, K., Inami, T., Ohwada, K., Yoshida, M., Jarrige, I., Tatami, N., Niioka, S., Bizen, D., Ando, Y., Mizuki, J., Maekawa, S. \& Endoh, Y. (2011). Phys. Rev. B, 83, 241101.

Ishii, K., Jarrige, I., Yoshida, M., Ikeuchi, K., Inami, T., Murakami, Y. \& Mizuki, J. (2013). J. Electron Spectrosc. Relat. Phenom. 188, 127132.

Jia, C. J., Nowadnick, E. A., Wohlfeld, K., Kung, Y. F., Chen, C.-C., Johnston, S., Tohyama, T., Moritz, B. \& Devereaux, T. P. (2014). Nat. Commun. 5, 3314.

Jia, C., Wohlfeld, K., Wang, Y., Moritz, B. \& Devereaux, T. P. (2016). Phys. Rev. X, 6, 021020.

Johann, H. H. (1931). Z. Phys. 69, 185-206.

Johansson, T. (1933). Z. Phys. 82, 507-528.

Ketenoglu, D., Harder, M., Klementiev, K., Upton, M., Taherkhani, M., Spiwek, M., Dill, F.-U., Wille, H.-C. \& Yavaş, H. (2015). J. Synchrotron Rad. 22, 961-967.

Kim, B. H. \& van den Brink, J. (2015). Phys. Rev. B, 92, 081105.

Kim, J., Casa, D., Upton, M. H., Gog, T., Kim, Y.-J., Mitchell, J. F., van Veenendaal, M., Daghofer, M., van den Brink, J., Khaliullin, G. \& Kim, B. J. (2012a). Phys. Rev. Lett. 108, 177003.

Kim, J., Daghofer, M., Said, A. H., Gog, T., van den Brink, J., Khaliullin, G. \& Kim, B. J. (2014). Nat. Commun. 5, 4453.

Kim, J., Said, A. H., Casa, D., Upton, M. H., Gog, T., Daghofer, M., Jackeli, G., van den Brink, J., Khaliullin, G. \& Kim, B. J. (2012b). Phys. Rev. Lett. 109, 157402.
Kim, Y.-J., Hill, J. P., Komiya, S., Ando, Y., Casa, D., Gog, T. \& Venkataraman, C. T. (2004). Phys. Rev. B, 70, 094524.

Kirkpatrick, P. \& Baez, A. V. (1948). J. Opt. Soc. Am. 38, 766-774.

Kotani, A. \& Shin, S. (2001). Rev. Mod. Phys. 73, 203-246.

Kvashnina, K. O. \& Scheinost, A. C. (2016). J. Synchrotron Rad. 23, 836-841.

Larson, B. C., Ku, W., Tischler, J. Z., Lee, C.-C., Restrepo, O. D., Eguiluz, A. G., Zschack, P. \& Finkelstein, K. D. (2007). Phys. Rev. Lett. 99, 026401.

Lee, W. S., Johnston, S., Moritz, B., Lee, J., Yi, M., Zhou, K. J., Schmitt, T., Patthey, L., Strocov, V., Kudo, K., Koike, Y., van den Brink, J., Devereaux, T. P. \& Shen, Z. X. (2013). Phys. Rev. Lett. 110, 265502.

Lee, W. S., Lee, J. J., Nowadnick, E. A., Gerber, S., Tabis, W., Huang, S. W., Strocov, V. N., Motoyama, E. M., Yu, G., Moritz, B., Huang, H. Y., Wang, R. P., Huang, Y. B., Wu, W. B., Chen, C. T., Huang, D. J., Greven, M., Schmitt, T., Shen, Z. X. \& Devereaux, T. P. (2014). Nat. Phys. 10, 883-889.

Le Tacon, M., Ghiringhelli, G., Chaloupka, J., Sala, M. M., Hinkov, V., Haverkort, M. W., Minola, M., Bakr, M., Zhou, K. J., BlancoCanosa, S., Monney, C., Song, Y. T., Sun, G. L., Lin, C. T., De Luca, G. M., Salluzzo, M., Khaliullin, G., Schmitt, T., Braicovich, L. \& Keimer, B. (2011). Nat. Phys. 7, 725-730.

Liu, X., Dean, M. P. M., Meng, Z. Y., Upton, M. H., Qi, T., Gog, T., Cao, Y., Lin, J. Q., Meyers, D., Ding, H., Cao, G. \& Hill, J. P. (2016). Phys. Rev. B, 93, 241102.

Llorens, I., Lahera, E., Delnet, W., Proux, O., Braillard, A., Hazemann, J.-L., Prat, A., Testemale, D., Dermigny, Q., Gelebart, F., Morand, M., Shukla, A., Bardou, N., Ulrich, O., Arnaud, S., Berar, J.-F., Boudet, N., Caillot, B., Chaurand, P., Rose, J., Doelsch, E., Martin, P. \& Solari, P. L. (2012). Rev. Sci. Instrum. 83, 063104.

Lu, L., Chabot-Couture, G., Zhao, X., Hancock, J. N., Kaneko, N., Vajk, O. P., Yu, G., Grenier, S., Kim, Y. J., Casa, D., Gog, T. \& Greven, M. (2005). Phys. Rev. Lett. 95, 217003.

Lu, X., McNally, D. E., Moretti Sala, M., Terzic, J., Upton, M. H., Casa, D., Ingold, G., Cao, G. \& Schmitt, T. (2017). Phys. Rev. Lett. 118, 027202.

Marra, P., Sykora, S., Wohlfeld, K. \& van den Brink, J. (2013). Phys. Rev. Lett. 110, 117005.

Marra, P., van den Brink, J. \& Sykora, S. (2016). Sci. Rep. 6, 25386.

Marra, P., Wohlfeld, K. \& van den Brink, J. (2012). Phys. Rev. Lett. 109, 117401.

Meyers, D., Miao, H., Walters, A. C., Bisogni, V., Springell, R. S., d'Astuto, M., Dantz, M., Pelliciari, J., Huang, H. Y., Okamoto, J., Huang, D. J., Hill, J. P., He, X., Božović, I., Schmitt, T. \& Dean, M. P. M. (2017). Phys. Rev. B, 95, 075139.

Minola, M., Dellea, G., Gretarsson, H., Peng, Y. Y., Lu, Y., Porras, J., Loew, T., Yakhou, F., Brookes, N. B., Huang, Y. B., Pelliciari, J., Schmitt, T., Ghiringhelli, G., Keimer, B., Braicovich, L. \& Le Tacon, M. (2015). Phys. Rev. Lett. 114, 217003.

Monney, C., Schmitt, T., Matt, C. E., Mesot, J., Strocov, V. N., Lipscombe, O. J., Hayden, S. M. \& Chang, J. (2016). Phys. Rev. B, 93, 075103.

Moretti Sala, M., Bisogni, V., Aruta, C., Balestrino, G., Berger, H., Brookes, N. B., de Luca, G. M., Di Castro, D., Grioni, M., Guarise, M., Medaglia, P. G., Miletto Granozio, F., Minola, M., Perna, P., Radovic, M., Salluzzo, M., Schmitt, T., Zhou, K. J., Braicovich, L. \& Ghiringhelli, G. (2011). New J. Phys. 13, 043026.

Moretti Sala, M., Boseggia, S., McMorrow, D. F. \& Monaco, G. (2014a). Phys. Rev. Lett. 112, 026403.

Moretti Sala, M., Henriquet, C., Simonelli, L., Verbeni, R. \& Monaco, G. (2013). J. Electron Spectrosc. Relat. Phenom. 188, 150-154.

Moretti Sala, M., Ohgushi, K., Al-Zein, A., Hirata, Y., Monaco, G. \& Krisch, M. (2014b). Phys. Rev. Lett. 112, 176402.

Moretti Sala, M., Schnells, V., Boseggia, S., Simonelli, L., Al-Zein, A., Vale, J. G., Paolasini, L., Hunter, E. C., Perry, R. S., Prabhakaran, D., Boothroyd, A. T., Krisch, M., Monaco, G., Rønnow, H. M., McMorrow, D. F. \& Mila, F. (2015). Phys. Rev. B, 92, 024405. 
Moser, S., Shaik, N. E., Samal, D., Fatale, S., Dalla Piazza, B., Dantz, M., Pelliciari, J., Olalde-Velasco, P., Schmitt, T., Koster, G., Mila, F., Rønnow, H. M. \& Grioni, M. (2015). Phys. Rev. B, 92, 140404.

Peng, Y. Y., Hashimoto, M., Sala, M. M., Amorese, A., Brookes, N. B., Dellea, G., Lee, W.-S., Minola, M., Schmitt, T., Yoshida, Y., Zhou, K.-J., Eisaki, H., Devereaux, T. P., Shen, Z.-X., Braicovich, L. \& Ghiringhelli, G. (2015). Phys. Rev. B, 92, 064517.

Ponchut, C., Rigal, J. M., Clément, J., Papillon, E., Homs, A. \& Petitdemange, S. (2011). J. Instrum. 6, C01069.

Rovezzi, M., Lapras, C., Manceau, A., Glatzel, P. \& Verbeni, R. (2017). Rev. Sci. Instrum. 88, 013108.

Shvyd'ko, Y., Hill, J., Burns, C., Coburn, D., Brajuskovic, B., Casa, D., Goetze, K., Gog, T., Khachatryan, R., Kim, J.-H., Kodituwakku, C., Ramanathan, M., Roberts, T., Said, A., Sinn, H., Shu, D., Stoupin, S., Upton, M., Wieczorek, M. \& Yavas, H. (2013). J. Electron Spectrosc. Relat. Phenom. 188, 140-149.

Shvyd'ko, Y. V., Lerche, M., Kuetgens, U., Rüter, H. D., Alatas, A. \& Zhao, J. (2006). Phys. Rev. Lett. 97, 235502.

Shvyd'ko, Y., Stoupin, S., Shu, D., Collins, S. P., Mundboth, K., Sutter, J. \& Tolkiehn, M. (2014). Nat. Commun. 5, 4219.

Shvyd'ko, Y., Stoupin, S., Shu, D. \& Khachatryan, R. (2011). Phys. Rev. A, 84, 053823.
Sokaras, D., Weng, T.-C., Nordlund, D., Alonso-Mori, R., Velikov, P., Wenger, D., Garachtchenko, A., George, M., Borzenets, V., Johnson, B., Rabedeau, T. \& Bergmann, U. (2013). Rev. Sci. Instrum. 84, 053102.

Suortti, P., Buslaps, T., Fajardo, P., Honkimäki, V., Kretzschmer, M., Lienert, U., McCarthy, J. E., Renier, M., Shukla, A., Tschentscher, T. \& Meinander, T. (1999). J. Synchrotron Rad. 6, 69-80.

Ulrich, C., Ament, L. J. P., Ghiringhelli, G., Braicovich, L., Moretti Sala, M., Pezzotta, N., Schmitt, T., Khaliullin, G., van den Brink, J., Roth, H., Lorenz, T. \& Keimer, B. (2009). Phys. Rev. Lett. 103, 107205.

Verbeni, R., Kocsis, M., Huotari, S., Krisch, M., Monaco, G., Sette, F. \& Vanko, G. (2005). J. Phys. Chem. Solids, 66, 22992305.

Warren, J. L., Yarnell, J. L., Dolling, G. \& Cowley, R. A. (1967). Phys. Rev. 158, 805-808.

Yavaş, H., Sutter, J. P., Gog, T., Wille, H.-C. \& Baron, A. Q. (2017). MRS Bull. 42, 424-429.

Yavaş, H., van Veenendaal, M., van den Brink, J., Ament, L. J. P., Alatas, A., Leu, B. M., Apostu, M.-O., Wizent, N., Behr, G., Sturhahn, W., Sinn, H. \& Alp, E. E. (2010). J. Phys. Condens. Matter, 22, 485601. 